\title{
Tools for $E_{6}$ model building
}

\author{
Gregory W. Anderson* \\ Department of Physics and Astronomy, Northwestern University \\ E-mail: 'ganderson@northwestern. edu'
}

ABstRACT: Recent progress in developing tools for $E_{6}$ model building is reported. These tools are used to illustrate a natural mechanism for Higgs mixing in $E_{6}$ models.

\section{Introduction}

The exceptional group $E_{6}$ has received consideration as a unified group for over twenty years [1], for the possibility that an entire generation of standard model fermions, a right handed neutrino and two Higgs doublet are unified into a single representation.

$$
\begin{aligned}
\mathbf{2 7} & =(\mathbf{3}, \mathbf{2})_{\mathbf{1}} \oplus(\overline{\mathbf{3}}, \mathbf{1})_{-\mathbf{4}} \\
& \oplus(\mathbf{3}, \mathbf{1})_{\mathbf{2}} \oplus(\overline{\mathbf{3}}, \mathbf{1})_{\mathbf{2}} \oplus(\overline{\mathbf{3}}, \mathbf{1})_{\mathbf{2}} \\
& \oplus(\mathbf{1}, \mathbf{2})_{-\mathbf{3}} \oplus(\mathbf{1}, \mathbf{2})_{-\mathbf{3}} \oplus(\mathbf{1}, \mathbf{2})_{\mathbf{3}} \\
& \oplus(\mathbf{1}, \mathbf{1})_{\mathbf{6}} \oplus(\mathbf{1}, \mathbf{1})_{\mathbf{0}} \oplus(\mathbf{1}, \mathbf{1})_{\mathbf{0}}
\end{aligned}
$$

Despite its potential, $E_{6}$ has been exploited to a much lesser extent than its subgroups $S U(5)$

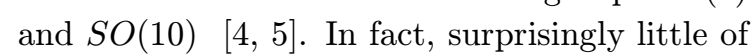
the group theory necessary to build a complete $E_{6}$ model had been explicitly worked prior to the work reported here [i, of the work by Koh, Patera and Rousseau [i]

The principle motivation for the work reported here [6. are currently being used to construct predictive models of fermion masses and mixing angles in $E_{6}$ unified theories [8], but our group theoretic results should be important and useful to anyone seeking to build an explicit unified models based on $E_{6}$.

\section{Model Building Considerations}

Although a wealth of predictive theories based on smaller groups like $S U(5)$ and $S O(10)$ have been

\footnotetext{
*Based on work in collaboration with Tomas Blazek
}

proposed, theories containing at most $S O(10)$ unification are in several respects unsatisfactory. First, from the point of view of supersymmetric theories, the distinction between the standard model fermions and the Higgs doublets as candidates for inclusion in a unified representation is artificial, since they are all contained in left handed chiral superfields. A supersymmetric extension of the one generation standard model would require the chiral superfields listed below:

$$
\begin{array}{ll}
S U(5): & \mathbf{1 0} \oplus \overline{\mathbf{5}} \oplus \overline{\mathbf{5}} \oplus \mathbf{5} \oplus \mathbf{1} \\
S O(10): & \mathbf{1 6} \oplus \mathbf{1 0} \\
E_{6}: & \mathbf{2 7}
\end{array}
$$

¿From this point of view, the unification present in $S U(5)$ and $S O(10)$ is seen to be incomplete.

Second, these theories do not naturally accommodate the fact that

$$
\frac{m_{d}}{m_{u}} \frac{m_{t}}{m_{b}} \gg 1
$$

without a loss of predictivity. Moreover, an $S O(10)$ model with a single 10 leads to the GUT scale relation $\lambda_{t}=\lambda_{b}=\lambda_{\tau}[\overline{9}]$, which implies $\tan \beta \sim$ $m_{t} / m_{b}$, which is problematic from the point of view of electroweak symmetry breaking. In order to avoid large $\tan \beta$ one must mix the Higgs doublets in the $\mathbf{1 0}$ with other doublet states. In the case where we have $n$ copies of the 10 dimensional representation, the mass matrix for the $n$ doublet pairs $H_{U}$ and $H_{D}$ induced at the GUT scale is an $n \times n$ matrix $\mu$ :

$$
-L \supset H_{U} \mu H_{D}
$$

In order to have a pair of doublets which do not acquire GUT scale masses we require $\operatorname{det} \mu=0$. 
The light $H_{U}\left(H_{D}\right)$ states are the zero eigenvectors of $\mu^{\dagger}(\mu)$ respectively. The most natural ways to accomplish this are to arrange family symmetries so that a row or column vanish, rows or columns proportional, or other symmetry properties which include, for example, a totally anti-symmetric matrix when $n=3$. The first and second cases are unnatural and lead to models with little predictivity while mass matrices typical of the latter cases:

$$
\mu \sim\left(\begin{array}{ccc} 
& X & \\
\pm X & Z & Y \\
\pm Y &
\end{array}\right), \mu \sim\left(\begin{array}{ccc} 
& A & B \\
-A & & C \\
-B & -C &
\end{array}\right)
$$

mix the $H_{U}$ and $H_{D}$ sectors identically leading to $\tan \beta \sim m_{t} / m_{b}$. As argued below, $E_{6}$ provides a natural solution to this problem.

\section{$E_{6}$ Clebsch Gordon coefficients}

In order to provide a complete construction of a supersymmetric $E_{6}$ theory, we have computed all of the Clebsch-Gordon coefficients occuring in in the renormalizable superpotential interactions of an $E_{6}$ theory containing fundamental, antifundamental and adjoint representations. The relevant renormalizable operators are listed below:

$$
\begin{array}{cll}
\text { Dimension } & \text { Singlets } & \\
2 & \mathbf{2 7} \cdot \overline{\mathbf{2 7}} & \{\mathbf{7 8}, \mathbf{7 8}\} \\
\multirow{2}{*}{3} & \{\mathbf{2 7}, \mathbf{2 7}\} \cdot \mathbf{2 7} & \{\overline{\mathbf{2 7}}, \overline{\mathbf{2 7}}\} \cdot \overline{\mathbf{2 7}} \\
& \mathbf{2 7} \cdot \overline{\mathbf{2 7}} \cdot \mathbf{7 8} & {[\mathbf{7 8}, \mathbf{7 8}] \mathbf{7 8}}
\end{array}
$$

In addition to the operators listed above, we have determined several of the dimension four operators in our analysis [i $\overline{6}$ ]. This calculation was made using standard techniques. The algebra is written as a set of ladder operators:

$$
\begin{aligned}
& {\left[H_{a}, H_{b}\right]=0} \\
& {\left[H_{a}, E_{ \pm \alpha_{i}}\right]= \pm \alpha_{i a} E_{ \pm \alpha_{i}}} \\
& {\left[E_{ \pm \alpha_{i}}, E_{ \pm \alpha_{j}}\right]= \begin{cases}0 & \alpha+\beta \text { not a root } \\
\alpha^{a} H_{a} & \alpha=-\beta \\
N_{\alpha \beta} E_{\alpha+\beta} & \text { otherwise }\end{cases} }
\end{aligned}
$$

States in a representation are obtained by a succession of lowering operators applied to the highest weight in the representation:

$$
\begin{aligned}
& \left(a_{1}, a_{2}, \ldots, a_{n}\right) \\
& E_{-\alpha_{j}} \quad a_{i}^{\prime}=a_{i}-A_{i j} \\
& \left(a_{1}^{\prime}, a_{2}^{\prime}, \ldots, a_{n}^{\prime}\right)
\end{aligned}
$$

The Dynkin coordinates of a weight $\mu$ : are $a_{i}=$ $2 \frac{\left\langle\mu, \alpha_{i}\right\rangle}{\left\langle\alpha_{i}, \alpha_{i}\right\rangle}$, and the Cartan matrix is:

$$
A_{i j}=2 \frac{\left\langle\alpha_{i}, \alpha_{j}\right\rangle}{\left\langle\alpha_{j}, \alpha_{j}\right\rangle}
$$

This procedure proves to be technically challenging for representations with a large number of degenerate weights. The lowering coefficients for degenerate weights are defined by:

$$
E_{ \pm \alpha_{i}}\left|\mu_{a}\right\rangle=N_{ \pm \alpha_{i}, \mu_{a} \rightarrow\left(\mu \pm \alpha_{i}\right)_{b}}\left|\left(\mu \pm \alpha_{i}\right)_{b}\right\rangle
$$

These lowering coefficients satisfy the recurrence relation:

$$
\begin{aligned}
& \left(G^{(\mu \pm \alpha)}\right)_{d c}^{-1} N_{ \pm \alpha, \mu_{a} \rightarrow(\mu \pm \alpha)_{c}} N_{ \pm \alpha, \mu_{b} \rightarrow(\mu \pm \alpha)_{d}}^{*} \\
& =\mp\left(G^{\mu}\right)_{b a}^{-1}\langle\alpha, \mu\rangle \\
& +G_{e f}^{(\mu \mp \alpha)}\left(G^{\mu}\right)_{b g}^{-1}\left(G^{\mu}\right)_{h a}^{-1} N_{ \pm \alpha(\mu \mp \alpha)_{e} \rightarrow \mu_{g}} \\
& \times N_{ \pm \alpha(\mu \mp \alpha)_{f} \rightarrow \mu_{h}}^{*}
\end{aligned}
$$

where the metric on the degenerate weight space is

$$
\begin{aligned}
& I_{\mu}=G_{i j}\left|\mu_{i}\right\rangle\left\langle\mu_{j}\right| \\
& G_{i j}=\left(M^{-1}\right)_{i j} \quad \text { where } \quad M_{i j}=\left\langle\mu_{i} \mid \mu_{j}\right\rangle .
\end{aligned}
$$

In general, lowering through a chain of degenerate weights involves three metrics:

$$
\begin{array}{cc}
\mu+\alpha & G^{(\mu+\alpha)} \\
\downarrow_{\mu} & E_{-\alpha} \\
\downarrow_{\mu-\alpha} & G_{-\alpha}^{(\mu)} \\
& G^{(\mu-\alpha)}
\end{array}
$$

The technical challenge is further aggravated by the fact that it is often impossible to choose 
a single basis for all of the states of a degenerate weight in a representation such that lowering a particular state results in a particular state of lower weight (as opposed to a linear combination of weights) for all possible lowerings.

The calculation of the product of two adjoint representation provides an example:

0

$1{ }_{(001000)}=\{(000001),(00100-1)\}$ ${ }_{(001000)}=\{(000001),(00100-1)\}_{ \pm}$ $2 \begin{cases}1 & 2430 \\ 1 & 2925\end{cases}$

6 $(100010)$ $\vdots$

11 (000001)

$$
22 \text { (оросо0) NB } 185 \rightarrow 36 \quad 108\left\{\begin{array}{cl}
36 & 2430 \\
45 & 2925 \\
20 & 650 \\
6 & 78 \\
1 & 1
\end{array}\right.
$$

For the degenerate (000000) weight, the 36 linearlyindependent degenerate weights of the $\mathbf{2 4 3 0}$ which occur at level 22 must be formed from 185 linearlydependent degenerate (000000) weights which one obtains after application of the lowering operator to the level 21 states.

\section{Masses and Mixings}

The relevant operators for the charged fermion masses and Higgs doublet mixing obtained from our analysis [i6], include a totally symmetric dimension three operator:

$$
O_{\{a b c\}}=27_{a} 27_{b} 27_{c},
$$

and two dimension-four operators which are respectively symmetric and anti-symmetric with respect to interchange of two fundamental representations

$$
\begin{aligned}
O_{\{a b\} c} & =\frac{1}{M}\left\{\mathbf{2 7}_{a}, \mathbf{2 7}_{b}\right\}\langle\mathbf{7 8}\rangle \mathbf{2} \mathbf{7}_{c} . \\
O_{[a b] c} & =\frac{1}{M}\left[\mathbf{2 7}_{a}, \mathbf{2 7}_{b}\right]\langle\mathbf{7 8}\rangle \mathbf{2} 7_{c},
\end{aligned}
$$

and Frogatt-Nielsen like [1] $\left.{ }_{0}^{\prime}\right]$ operators which can be generated by integrating heavy vector-like $\mathbf{2 7}-\overline{\mathbf{2 7}}$ pairs out of the theory:

$$
O_{a b c}={ }_{27} \frac{\langle\mathbf{7 8}\rangle_{1}}{\langle 1,78\rangle_{1}} \cdots \frac{\langle\mathbf{7 8}\rangle_{n}}{\langle\mathbf{1 , 7 8}\rangle_{n}} \mathbf{2 7 _ { b }} \frac{\langle\mathbf{7 8}\rangle_{n+1}}{\langle\mathbf{1 , 7 8}\rangle_{\mathbf{n}+1}} \cdots \frac{\langle\mathbf{7 8}\rangle_{l}}{\langle 1,78\rangle_{l}} \mathbf{2 7 _ { c }}
$$

These operators, like the analogous operators for $S O(10)\left[\begin{array}{l}1 \\ 1\end{array}\right]$, relate the Yukawa couplings $Y_{u, d, e}$ up to possible Clebsch factors from vevs of the adjoint representation. The larger unification in $E_{6}$ gives an additional relation between these couplings, the neutrino Yukawa couplings, and the mixing of the Higgs doublets. Below we list example induced mixings, for $\left\{\mathbf{2 7}_{i}, \mathbf{2 7}_{j}\right\}_{ \pm} \mathbf{2 7}$ : induced by the operators above:

$$
\begin{aligned}
Y_{F} & =\left(\begin{array}{ccc}
0 & C_{F^{c}}^{ \pm} H_{k} & \pm C_{F^{c}}^{ \pm} H_{j} \\
C_{F}^{ \pm} H_{k} & 0 & \pm C_{\phi}^{ \pm} H_{i} \\
\pm C_{F}^{ \pm} H_{j} & C_{\phi}^{ \pm} H_{i} & 0
\end{array}\right) \\
\mu & =\left(\begin{array}{ccc}
0 & C_{H}^{ \pm}\left\langle S_{k}\right\rangle & \pm C_{H}^{ \pm}\left\langle S_{j}\right\rangle \\
C_{h}^{ \pm}\left\langle S_{k}\right\rangle & 0 & \pm C_{S}^{ \pm}\left\langle S_{i}\right\rangle \\
\pm C_{h}^{ \pm}\left\langle S_{j}\right\rangle & C_{S}^{ \pm}\left\langle S_{i}\right\rangle & 0
\end{array}\right)
\end{aligned}
$$

the anti-symmetric operator is seen to provide a natural solution to $\operatorname{det} \mu=0$, with unequal mixings for $H_{U}$ and $H_{D}$ as advertised.

\section{Acknowledgements}

This work was supported in part by Northwestern University and the U.S. Department of Energy, Division of High Energy Physics, under Grant No. DE-FG02-91-ER4086.

\section{References}

[1] F. Gürsey, P. Ramond, and P. Sikivie, Phys. Lett. B60 (1976) 177; 
[2] Y. Achiman, B. Stech, Phys. Lett. B77 (1978) 389; F. Gürsey, and M. Serdaroğlu, Nuovo Cimento 65A (1981) 337.

[3] R. Slansky, Phys. Rep. 79 (1981) 1.

[4] H. Georgi and S. Glashow, Phys. Rev. Lett. 32 438 (1974);

[5] H. Georgi, in Proceedings of the American Institute of Physics, at William and Mary College (1974); H. Fritch, and P. Minkowsi, Ann. Phys. 93, 193 (1975); M. Gell-Mann, P. Ramond, R. Slansky, Rev. Mod. Phys. 50, 721 (1978).

[6] G. W. Anderson and T. Blazek, hep-ph/9912365, to appear in J. Math. Phys; hep-ph/0006017 to appear in J. Math. Phys.

[7] In-Guy Koh, J. Patera, and C. Rousseau, J. Math. Phys. 25 (1984) 2863.

[8] G. W. Anderson and T. Blazek, "Exceptional Models of Fermion Masses and Mixing Angles.," NUUEP-TH-2000-90 (Manuscript' - . - in preparation),

[9] B. Ananthanarayan, G. Lazarides and Q. Shafi, Phys. Rev. D44 1613 (1991); H. Arason, D. Castano, B. Keszthelyi, S. Mikaelian, E. J. Piard, P. Ramond and B. Wright, Phys. Rev. Lett. 67 2933 (1991).

[10] C. D. Froggatt and H. B. Nielsen, Nucl. Phys. B147 277 (1979).

[11] G. Anderson, S. Dimopoulos, L. Hall, S. Raby, and G. Starkman, Phys. Rev. D49, 3660-3690 (1994). 